\title{
The relationship of glycaemic level to advanced glycation end-product (AGE) accumulation and retinal pathology in the spontaneous diabetic hamster
}

\author{
H.-P.Hammes ${ }^{1}$, B. Wellensiek ${ }^{1}$, I. Klöting ${ }^{2}$, E. Sickel ${ }^{3}$, R. G. Bretzel ${ }^{1}$, M. Brownlee ${ }^{4}$ \\ ${ }^{1}$ Third Medical Department, Justus-Liebig-University of Giessen, Germany \\ ${ }^{2}$ Diabetes Research Institute, Karlsburg, Germany \\ ${ }^{3}$ Central Institute for Laboratory Breeding, Hannover, Germany \\ ${ }^{4}$ Diabetes Research Center and Departments of Medicine and Pathology, Albert Einstein College of Medicine, Bronx, \\ New York, USA
}

\begin{abstract}
Summary To assess the relationship between glucose and advanced glycation end products (AGE) and the relationship between $\mathrm{AGE}$ and retinal changes in vivo, we studied the time course of retinopathy over 12 months in trypsin digest preparations and measured glycaemia and retinal AGE in spontaneous diabetic hamsters of mild (MD) and severe (SD) phenotypes. Blood glucose levels were elevated in MD $(9.44 \pm 0.76 \mathrm{mmol} / \mathrm{l})$ and in SD (3 months: $24.3 \pm$ $1.4 \mathrm{mmol} / \mathrm{l} ; 12$ months: $31.7 \pm 0.8 \mathrm{mmol} / \mathrm{l})$ over nondiabetic controls (NC: $7.15 \pm 0.25 \mathrm{mmol} / 1 ; p<0.05$ or less vs $\mathrm{MD} ; p<0.001$ vs $\mathrm{SD})$. Similar relations were found for $\mathrm{HbA}_{1}$. Retinal AGE in mild diabetes was $405 \pm 11.3$ arbitrary units (AU) (NC 245 \pm 7.7 ; $p<0.01$ ) after 3 months and remained unchanged. A non-linear increase of AGE over time was found in severe hyperglycaemic hamsters $(466 \pm 21$ AU after 3 months and $758 \pm 21$ AU after 12 months; $p<0.001$ vs MD). Pericyte loss in mild diabetes progressed from $-26 \%$ after 3 months to $-41 \%$ after 12 months $(p<0.001$ vs NC). Whereas the initial pericyte loss in
\end{abstract}

severely diabetic hamsters was identical to the mildly diabetic group, a higher degree of pericyte loss occurred after 12 months $(-57 \% ; p<0.05$ vs MD). Endothelial cell numbers remained unaffected by mild hyperglycaemia, but significantly increased over time in severe diabetes reaching $31.7 \%$ above controls after 12 months $(p<0.001$ vs NC and MD). Microaneurysms were absent in all retinae examined. Acellular capillary segments were increased in mild diabetes $\left(3.83 \pm 0.31\right.$ per $\mathrm{mm}^{2}$ of retinal area) and severe diabetes $(7.83 \pm 0.73)$ over controls $(1.0 \pm 0.23)$. These data suggest that a threshold of glycaemia might exist above which AGE removal systems become saturated. Pericyte loss and acellular capillary formation are associated with mild increases in blood glucose and AGE levels while endothelial cell proliferation requires higher glucose and AGE levels. [Diabetologia (1998) 41: 165-170]

Keywords Diabetic retinopathy, chinese hamster, advanced-glycation end-products, pericytes.
The most characteristic findings of diabetic background retinopathy in humans are increased capillary permeability and progressive vascular occlusions. According to studies correlating angiographic in vivo findings with post-mortem retinal digests microaneurysms cluster around areas of capillary

Received: 31 July 1997 and in revised form: 29 September 1997

Corresponding author: Dr. H.-P. Hammes, Third Medical Department, Justus Liebig University, Rodthohl 6, D-35392 Giessen, Germany

Abbreviations: AGE, Advanced glycation end products; VEGF, vascular endothelial growth factor. non-perfusion [1, 2], possibly in an early abortive attempt at retinal new vessel formation. From earlier work it is known that pericytes may disappear leaving behind empty pockets in the capillary basement membrane [3]. Since endothelial cells do not leave behind such traces when they disappear, their fate during the initial course of retinal damage is uncertain, despite indication for both focal loss, leading to acellular capillaries, and focal proliferations leading to hypercellular vessels and microaneurysms [4, 5]. Pericyte loss and acellular, occluded capillaries have been found in diabetic animals, in which the natural course of diabetic retinopathy has been studied $[6,7]$. 
Results of the Diabetes Control and Complications Trial (DCCT) established the causal role of chronic hyperglycaemia in the pathogenesis of diabetic microvascular complications [8]. With higher levels of glycaemia the percentage of onset and progression of diabetic retinopathy increased. However, the exact sequence of retinal changes and the biochemical mechanisms underlying hyperglycaemia-associated retinal changes are still unclear.

One mechanism involved is the formation and accumulation of advanced glycation end products (AGE) [9]. We have previously shown that the administration of aminoguanidine, an inhibitor of AGE formation, results both in a significant attenuation of extracellular AGE accumulation in retinal vascular basement membranes and in an almost $80 \%$ reduction of acellular capillaries of diabetic rats [10]. Despite this indication of a major role of AGE in the pathogenesis of diabetic retinopathy, the relationship between glucose level and AGE level in vivo and the relationship between AGE level and retinal changes in diabetes are unknown.

Therefore, the aim of the present study was to investigate in a time course the retinal changes of diabetic animals with different glycaemic levels and to assess in vivo the relation between these changes with the degree of hyperglycaemia and the level of retinal extracellular autofluorescent AGE. We chose a spontaneous diabetic hamster model, in which two sublines of different glycaemic levels have been created through breeding, one with mild and one with severe hyperglycaemia [11]. Previous studies established that the spontaneous diabetic hamster eventually develops some early features of diabetic retinopathy such as acellular capillaries and an increase in the capillary endothelial cell to pericyte ratio [12]. Using retinal digest preparations of animals with various durations of diabetes (3-12 months) we compared animals with mild and severe hyperglycaemia, measured retinal autofluorescent AGE and parameters of diabetic retinopathy such as pericyte loss and acellular capillaries.

\section{Materials and methods}

Animals. The use of animals in this study conformed to the Association for Research in Vision and Ophthalmology Resolution on the Use of Animals in Research.

Sublines of the Chinese hamster (Crisetulus griseus) were used in this study. Depending on the parental phenotype, animals were severely diabetic (SD group) with plasma glucose values exceeding $15 \mathrm{mmol} / \mathrm{l}$ at manifestation with a continuous increase up to $30 \mathrm{mmol} / \mathrm{l}$ over time, and were glucosuric. Mildly diabetic hamsters (MD) were defined as having fluctuating plasma glucose values between 5 and $16 \mathrm{mmol} / \mathrm{l}$ without significant glucosuria. The non-diabetic Han-CHIA subline served as controls (NC). A detailed description of the animals has been given elsewhere [11].
Animals were kept under standardized conventional holding conditions regarding temperature and light cycles and had free access to water and standard laboratory chow.

Blood glucose was measured at regular intervals using a standard glucose oxidase method. The glycated haemoglobin of each animal was measured at the end of the study using an affinity chromatography method (Glyc Affin; Isolab, Akron, Ohio, USA).

At the end of each study period (3, 6, 9, and 12 months), animals were killed under deep ether anaesthesia and eyes were removed and fixed in $4 \%$ buffered formalin. In the control group, 7, 3, 3, and 6 retinae were available for analysis after 3 , 6,9 , and 12 months. In the mildly diabetic group, the numbers were $5,2,6$, and 6 , and in the severely diabetic group, the numbers were $8,9,7$, and 4 animals per group.

Retinal digest preparations. After retinal isolation, the samples were washed in distilled water for $75 \mathrm{~min}$. A combined digestion $-5 \%$ pepsin in $0.2 \%$ hydrochloric acid for $1 \mathrm{~h}$, then $3 \%$ trypsin in $0.2 \mathrm{~mol} / 1$ Tris for $3.5 \mathrm{~h}$ - was used to isolate the retinal vessel system. The preparations were placed on glass slides, washed with distilled water, air-dried and stained with periodic acid/Schiff reagent (PAS)/haematoxylin. One retina per animal was used for morphometric evaluation.

AGEs and the numbers of acellular capillary segments were measured as described previously [13]. Retinae were completely scored for the presence of acellular vessels (integration ocular Olympus, $400 \times$ magnification). Only completely acellular capillaries were recorded as positive, and values are given as numbers of acellular capillary segments per $\mathrm{mm}^{2}$ of retinal area. Moreover, the complete retinae were scored for the presence of microaneurysms.

The numbers of endothelial cells and pericytes were counted in 10 randomly selected fields of each retina by using the image analysing system with a morphometric software program (CUE 2; Olympus Opticals Europe, Hamburg, Germany) and the total for each retina was expressed as mol $\mu \mathrm{m}^{2}$ of the capillary area.

All morphometric evaluations were performed by two observers unaware of the identity of the samples being examined.

Statistical analysis. All parameters are given as mean \pm SEM. For statistical analysis, ANOVA and the Bonferroni multiple comparison test (Instat; GraphPad, San Diego, Calif., USA) were used.

\section{Results}

Blood glucose values of the three groups examined are given in Figure $1 \mathrm{a}$. The mean random glucose level of the non-diabetic subline (NC; CHIA) was $7.15 \pm 0.25 \mathrm{mmol} / \mathrm{l}$ throughout the study. Concomittantly, in this group body weight increased from $36.4 \pm 2.2 \mathrm{~g}$ after 3 months to $47.0 \pm 4 \mathrm{~g}$ at 12 months. Random blood glucose values of the mildly diabetic subline (MD; CHIG) significantly differed from the control group at all time points examined at an average of $9.44 \pm 0.76 \mathrm{mmol} / 1$ ( $p<0.001 \mathrm{vs} \mathrm{NC})$. In parallel with the rise in glycaemia, body weight in mildly diabetic hamsters remained unchanged $(36.0 \pm 3.8 \mathrm{~g}$ after 3 months; $38.7 \pm 6.1 \mathrm{~g}$ after 12 months). Severely diabetic hamsters (SD; CHIG) were characterized by a 3.7-fold increase in mean blood glucose, compared 

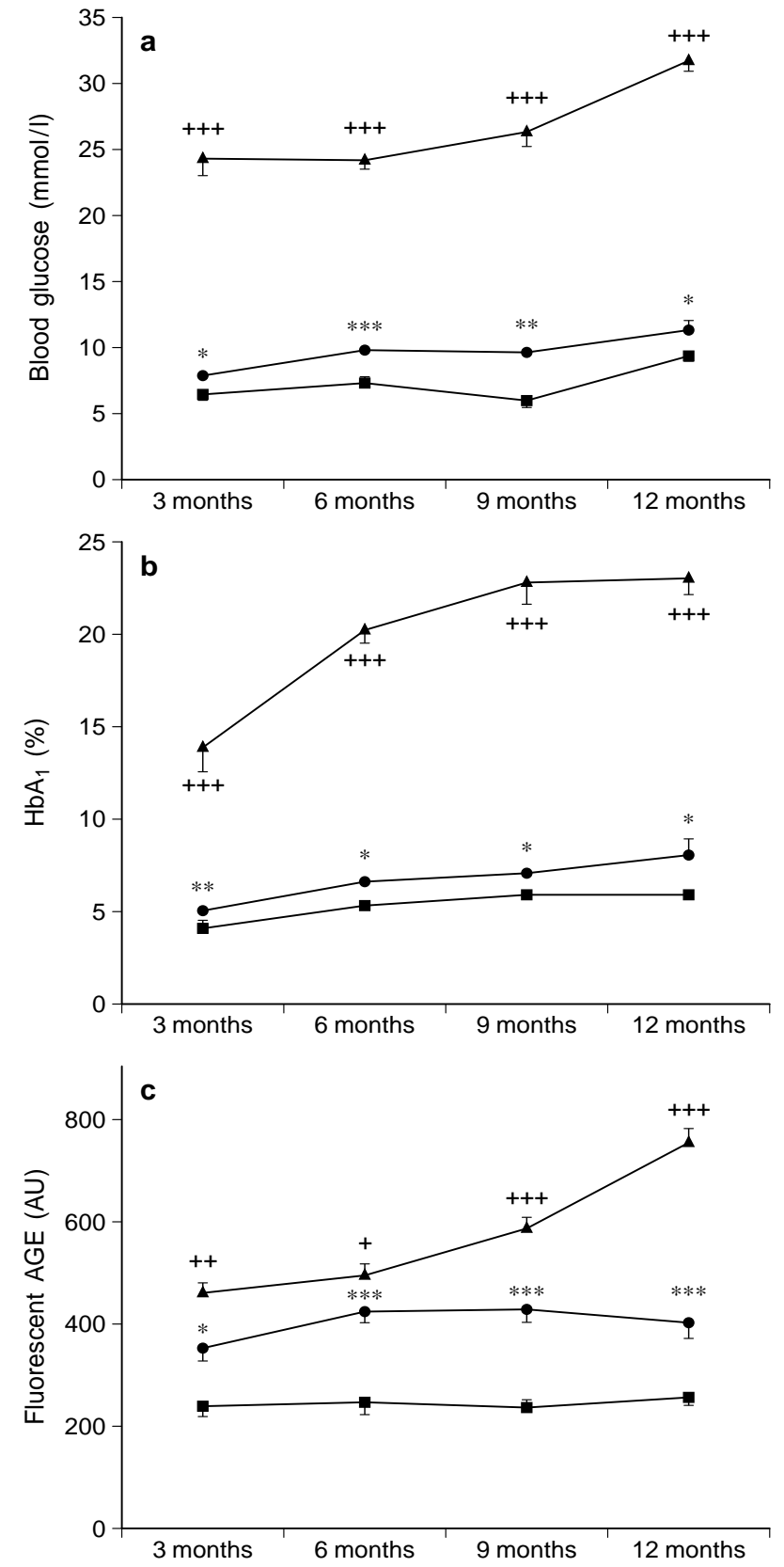

Fig.1a-c. Random blood glucose (a), glycated haemoglobin (b), and retinal AGE (c) in mildly (MD, ) and severely (SD, $\Delta)$ diabetic hamsters, compared to non-diabetic control animals (NC, $\square$ ). Mean \pm SEM; * **, *** $p<0.05,0.01,0.001$ vs $\mathrm{NC} ;+++p<0.001$ vs MD)

with the control group, accompanied by a small loss of average weight ( 3 months: $35.8 \pm 3.6 \mathrm{~g} ; 12$ months $33.2 \pm 3.2 \mathrm{~g})$.

Mean blood glucose levels, as measured by haemoglobin $\mathrm{A}_{1}$, were consistently higher in the mildly diabetic and even much higher in the severely diabetic group, reflecting and verifying the different glycaemic levels in the groups examined (Fig. $1 \mathrm{~b}$ ).

Next, we studied the levels of extracellular fluorescent AGE, deposited in precapillary arteriolar

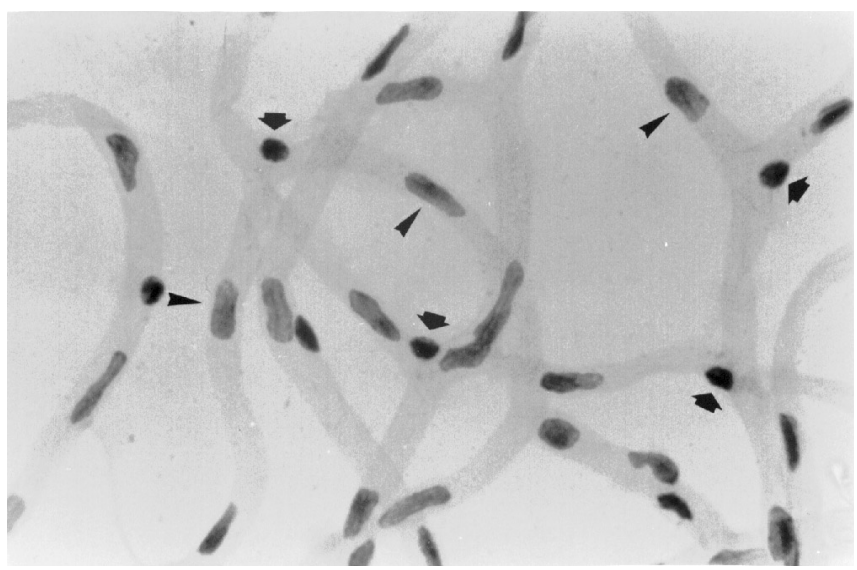

Fig. 2. Photomicrograph of a retinal digest preparation from a 12 month old non-diabetic hamster. Note the difference between nuclei of endothelial cells (marked by arrowheads; pale, elongated) and pericytes (marked by arrows; dark, small, round). PAS-staining, original magnification $250 \times$

branching sites of the retinal vasculature. Fluorescent AGE measurements revealed a significant increase in the mildly diabetic group, indicating that slightly elevated blood glucose levels can result in a significant increase in AGE formation. On average, fluorescent AGE in the control group were $245 \pm 7.7$ arbitrary units (AU) and $405 \pm 11.3$ AU in the mildly diabetic group $(p<0.001)$. In contrast to the known properties of AGE in vitro, the relative increase in AGE in mildly diabetic animals was higher than expected from their mean glucose levels. The lack of a further increase despite the cumulative character of AGE formation is consistent with the turnover of proteins. In severely diabetic hamsters, there was not only a significantly increased AGE deposition in retinal matrix where AGE were measured, compared to mildly diabetic animals, but also a non-linear increase over the study period, reaching a 3-fold level after 12 months compared with the age-matched control group (Fig. 1c). The continued increase in AGE is consistent with changes in protein turnover.

Morphometric analysis of retinal digest preparations was greatly facilitated by the excellent distinction of endothelial cells from pericytes in this species (Fig. 2). Thus, the numbers of cells which could not unequivocally be assigned to one of the two cell types, were virtually negative.

Endothelial cell counts in non-diabetic hamsters averaged $2160 \pm 40.3$ cells $/ \mathrm{mm}^{2}$ of capillary area and slightly decreased over time. In mildly diabetic hamsters, endothelial cell numbers showed an insignificant, small increase with a concomitant decrease over time. In the severely diabetic group, a $15 \%$ increase of endothelial cells was already present at the 3 month examination point and progressively increased over time, reaching a $31.7 \%$ increase after 

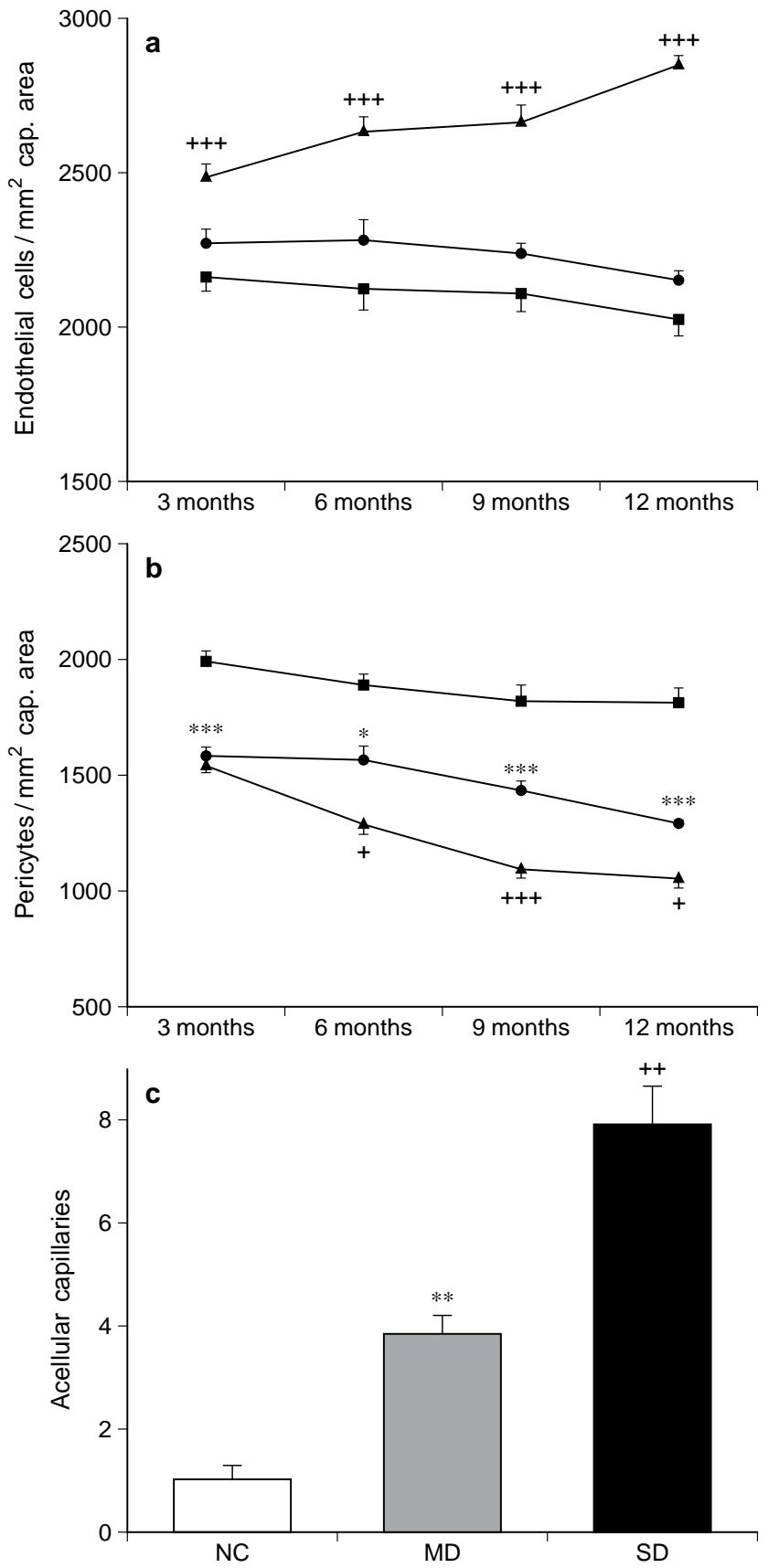

Fig.3a-c. Time course of endothelial cell (a) and pericyte numbers (b) in mildly (O) and severely diabetic $(\boldsymbol{\Delta})$ hamsters compared to non-diabetic controls $(\square)$. (c) Acellular capillary segments of 12 month diabetic and age-matched control hamsters. $++,+++p<0.01,0.001$ vs MD; *,**,*** $p<0.05$, $0.01,0.001$ vs NC

12 months compared with the control group $\left(2843 \pm 38\right.$ endothelial cells $\left./ \mathrm{mm}^{2}\right)$ (Fig. $3 \mathrm{a}$ ).

The numbers of pericytes did not show any significant change during the study period in non-diabetic hamsters, although a slight trend towards a decrease was observed (Fig. 3 b). In mildly hyperglycaemic animals, a highly significant pericyte loss was already present after only 3 months and slightly progressed over time (MD 3 months $1570 \pm 44$ pericytes $/ \mathrm{mm}^{2}$ vs MD 12 months $1270 \pm 75$ pericytes $/ \mathrm{mm}^{2} ; p<0.001$ ). Interestingly, the degree of pericyte loss in severely diabetic hamsters was identical to that of the mildly diabetic group after 3 months. Thereafter, a progressive dropout occurred reaching $57.7 \%$ of the numbers in the non-diabetic hamsters after 12 months.

Acellular capillaries were not detected in animals with a study duration of less than 12 months. Only in the animals which were followed for 12 months were the numbers of acellular capillary segments increased. Mildly diabetic hamsters had $3.83 \pm 0.31$ acellular capillary segments $/ \mathrm{mm}^{2}$, and severely diabetic animals had $7.83 \pm 0.73$ acellular capillary segments $/ \mathrm{mm}^{2}$ of retinal area ( $p<0.001$ between groups) (Fig. 3c). Non-diabetic animals had $1.0 \pm 0.28$ acellular capillary segments. No microaneurysms were found in any of the animals studied.

\section{Discussion}

The main findings of the present study indicate:

1) a threshold of glycaemia exists above which AGE removal systems become saturated;

2) pericyte loss and acellular capillary formation are associated with increased hyperglycaemia and AGE levels; and

3 ) endothelial cell proliferation requires higher glucose levels than pericyte loss.

Retinal morphometry in the hamster, based on the excellent differentiability of capillary endothelial cells from pericytes, shows that pericyte loss is the first measurable change in retinal capillaries, and that acellular capillaries as the most significant early pathological lesion in diabetic retinopathy occur only after a prolonged period of time. The individual ability of the two capillary cell types to compensate for hyperglycaemia-induced cell injury or even death [14] appears to be different. While pericytes are lost, endothelial cells proliferate. The degree of proliferation appears not to be determined by the degree of pericyte dropout, as suggested earlier [15]. Since our in vivo data show that despite an identical degree of pericyte loss in both diabetic groups after 3 months of diabetes, endothelial cells were only increased in the severely diabetic group, the degree of hyperglycaemia might preferentially determine the level of endothelial cell rather than the degree of pericyte loss. Hyperglycaemia per se induces increased gene transcription of basic fibroblast growth factor and vascular endothelial growth factor (VEGF) in cell culture $[16,17]$. AGE can upregulate VEGF mRNA in vitro and upon intravitreal injection in vivo induce microaneurysms and other characteristic lesions of background retinopathy $[18,19]$. Supporting that 
AGE induce endothelial cell proliferation, long-term treatment of diabetic rats with the AGE-inhibitor aminoguanidine completely abrogates endothelial cell proliferation [10]. An increased production of reactive oxygen intermediates as a result of glucose autoxidation, autoxidation of glycated proteins and AGE-binding to cellular receptors can also upregulate VEGF [20-23].

A plateau of autofluorescent AGE, significantly elevated over non-diabetic control levels, was determined after 3 months of diabetes as the first in vivo evidence that small increases of glucose lead to significant increases in AGE. The plateau represents a steady-state in which increased glucose levels induce higher formation of AGE which equals the rate of degradation. The significant increase of AGE in mild diabetes is consistent with in vitro data showing that AGE modify the turnover of affected proteins $[24,25]$. Both long-lived matrix molecules such as basement-membrane constituents, and plasma proteins, extravasated through hyperglycaemiamediated increased vascular leakage, are rendered less susceptible to proteolytic degradation through AGE-modification. Our observations are also consistent with the upregulation of endogenous enzymes which limit the formation and subsequent accumulation of AGE. As one example, an aldehyde reductase has been described which reduces reactive AGE intermediates (for example 3-deoxyglucosone) to less reactive compounds (3-deoxyfructose) [26]. Severe diabetes where AGE formation keeps increasing, represents a state where glucose-induced formation is greater than the rate of removal which is consistent with the saturation of the removal systems.

In this study, mild hyperglycaemia was associated with acellular capillaries only in the group of hamsters with the longest observation period. The significant increase in severely diabetic animals indicates that the degree of glycaemia determines the extent of capillary closure. Endothelial cell loss which determines the occurrence of acellular capillaries in capillaries where pericytes are lost, is the likely result of both the accumulation of extracellular AGE and the dysregulation in locally available growth-promoting cytokines [27, 28].

In conclusion, this study examines for the first time in vivo in a time course the relation between glycaemia, AGE and retinal lesions in a spontaneously diabetic animal. Although translation of these results into human diabetes is difficult, the identification of factors mediating differential response of retinal capillary cells to permanent hyperglycaemic injury may be useful for therapeutic targeting. Whether unravelling the determinants of AGE formation/elimination and possible defects may provide an approach to identify high risk individuals with the prospect of early treatment with AGE-inhibitors remains open.
Acknowledgements. The excellent technical assistance of Antje Weiss is greatly appreciated. This study was partly supported by grant Ha 1755/1-3 from the German Research Council, Bonn, Germany.

This work is part of the doctoral thesis of B. W. at the Faculty of Veterinary Medicine, Justus-Liebig-University, Giessen, Germany.

\section{References}

1. Kohner EM, Henkind P (1970) Correlation of fluorescein angiogram and retinal digest in diabetic retinopathy. Am J Ophthalmol 69: 403-414

2. Bresnick GH, Davis MD, Myers FL, de Venecia G (1977) Clinicopathologic correlations in diabetic retinopathy. II. Clinical and histologic appearances of retinal capillary microaneurysms. Arch Ophthalmol 95(7):1215-1220

3. Cogan DG, Toussaint D, Kuwabara T (1961) Retinal vascular pattern. IV. Diabetic retinopathy. Arch Ophthalmol 66: 366-378

4. Kuwabara T, Cogan DG (1965) Retinal vascular patterns. VII. Acellular change. Invest Ophthalmol 4(6):1049-1064

5. Bresnick GH, Engerman R, Davis MD, de-Venecia-G, Myers FL (1976) Patterns of ischemia in diabetic retinopathy. Trans Am Acad Ophthalmol Otolaryngol 81: 694-709

6. Engerman RL, Kern TS (1995) Retinopathy in animal models of diabetes. Diabetes Metab Rev 11: 109-120

7. Hammes HP, Syed-Ali S, Uhlmann M, et al. (1995) Aminoguanidine does not inhibit the initial phase of experimental diabetic retinopathy in rats. Diabetologia 38: 269-273

8. DCCT Research Group (1993) The effect of intensive treatment of diabetes on the development and progression of long-term complications in insulin-dependent diabetes mellitus. N Engl J Med 329: 977-986

9. Brownlee M (1994) Lilly lecture 1993. Glycation and diabetic complications. Diabetes 43: 836-841

10. Hammes HP, Martin S, Federlin K, Geisen K, Brownlee M (1991) Aminoguanidine treatment inhibits the development of experimental diabetic retinopathy. Proc Natl Acad Sci USA 88: 11555-11558

11. Klöting I, Sickel E, Vogt L, Kohnert KD (1994) CHIG-Han hamsters: an animal model for diabetes research. In: Bunyan B (ed) Proceedings of the 5th FELASA Symposium, Royal Society of Medicine Press, London, pp 190-194

12. Gerritsen GC (1982) The Chinese hamster as a model for the study of diabetes mellitus. Diabetes 31 [Suppl 1]:14-23

13. Hammes HP, Strödter D, Weiss A, Bretzel, RG, Federlin K, Brownlee M (1995) Secondary intervention with aminoguanidine retards the progression of diabetic retinopathy in the rat model. Diabetologia 38: 656-660

14. Mitsutani M, Kern TS, Lorenzi M (1996) Accelerated death of retinal microvascular cells in human and experimental diabetic retinopathy. J Clin Invest 97: 2883-2890

15. Antonelli-Orlidge A, Saunders KB, Smith SR, D'Amore PA (1989) An activated form of transforming growth factor beta is produced by cocultures of endothelial cells and pericytes. Proc Natl Acad Sci USA 86: 4544-4548

16. McClain DA, Paterson AJ, Roos MD, Wie X, Kudlow JEO (1992) Glucose and glucosamine regulate growth factor gene expression in vascular smooth muscle cells. Proc Natl Acad Sci USA 89: 8150-8154

17. Sone H, Kawakami Y, Okuda Y, et al. (1996) Vascular endothelial growth factor is induced by long-term high glucose concentration and up-regulation by acute glucose de- 
privation in cultured bovine retinal pigmented epithelial cells. Biochem Biophys Res Commun 221: 193-198

18. Yamagishi S, Yonekura H, Yamamoto Y, et al. (1997) Advanced glycation end products-driven angiogenesis in vitro. J Biol Chem 272: 8723-8730

19. Tolentino MJ, Miller JW, Gradoudas ES, et al. (1996) Intravitreous injections of vascular endothelial growth factor produce retinal ischemia and microangiopathy in an adult primate. Ophthalmology 103: 1820-1828

20. Hunt JV, Wolff SP (1991) Oxidative glycation and free radical production: a causal mechanism of diabetic complications. Free Radic Res Commun 12-13: 115-123

21. Mullarkey CJ, Edelstein D, Brownlee M (1990) Free radical generation by early glycation products: a mechanism for accelerated atherogenesis in diabetes. Biochem Biophys Res Commun 173: 932-939

22. Yan SD, Schmidt AM, Anderson GM, et al. (1994) Enhanced cellular oxidant stress by the interaction of advanced glycation end products with their receptors/binding proteins. J Biol Chem 269: 9889-9897

23. Kuroki M, Voest EE, Amano S, et al. (1996) Reactive oxygen intermediates increase vascular endothelial growth fac- tor expression in vitro and in vivo. J Clin Invest 98: 16671675

24. Brownlee M, Pongor S, Cerami A (1983) Covalent attachment of soluble proteins by nonenzymatically glycosylated collagen: role in the in situ formation of immune complexes. J Exp Med 158: 1739-1744

25. Lubec G, Pollack A (1980) Reduced susceptibility of nonenzymatically glycosylated glomerular basement membrane to proteases. Renal Physiol 3: 4-8

26. Takahashi M, Fujii J, Teshima T, Suzuki K, Shiba T, Taniguchi N (1993) Identity of a major 3-deoxyglucosone-reducing enzyme with aldehyde reductase in rat liver established by amino acid sequencing and cDNA expression. Gene 127: 249-253

27. Haitoglou CS, Tsilibary EC, Brownlee M, Charonis AS (1992) Altered cellular interactions between endothelial cells and nonenzymatically glucosylated laminin/type IV collagen. J Biol Chem 267: 12404-12407

28. Hammes HP, Weiss A, Hess S, et al. (1996) Modification of vitronectin by advanced glycation alters functional properties in vitro and in the diabetic retina. Lab Invest 75: 325 338 